\title{
A Framework for Dynamical True Altitude Modeling in Aviation
}

\author{
Josaphat A. Uvah \\ University of West Florida, Pensacola, USA
}

\begin{abstract}
In this paper, we examine some of the mathematical models for determining true altitude that are currently in use in aviation. The International Standard Atmosphere (ISA) is the prevalent model in current use. Moreover, several other models emanate from the ISA. Thus, we identify the basic physical laws as well as the model assumptions utilized to develop the ISA. Aside from highlighting the main limitations in the theoretical construction of the ISA, we identify some challenges encountered in its implementation in specific dynamical aviation settings where external input from air navigation systems is unavailable. Using Charles' Law and the hypsometric equation, we formulate a new and innovative theoretical basis for dynamical modeling of the true altitude as is applicable to aviation.
\end{abstract}

Keywords: true altitude, mathematical modeling, aviation, International Standard Atmosphere (ISA)

\section{Introduction}

True altitude, also known variously as geometric altitude or tapeline, is a central measurement in many aviation operations. Succinctly defined, it is the height of an object, such as an aircraft above mean sea level as determined by a physical tape measure (Olson, 2002). In modern aviation settings involving complex and specialized operations, where external inputs are unavailable to the aircraft crew but precision remains of the essence, the importance of true altitude assumes a more pronounced dimension in the safety of the operation. A typical example is a military flight in hostile territory where airport tower help is neither sought nor anticipated, radars are hopelessly jammed for obvious reasons, and normal external inputs are simply unavailable. Similarly, when a civilian aircraft is in the vicinity of an isolated airfield in extreme weather, contact with external guides may become severely hampered and the aircraft crew members must depend on themselves for a period of time with on-board resources exclusively. Traditional methods for computing true altitude depend heavily on a mathematical model, such as the International Standard Atmosphere (ISA) as well as external inputs from airport towers and navigation systems, such as the global positioning system (GPS). Essentially, the model is utilized to calibrate an instrument - the barometric altimeter, whose readings are used to approximate true altitude. This traditional method continues to serve its purpose for regular flight operations under normal conditions, because it provides for a sufficiently safe and effective operation.

In recent decades, advances in technology have enhanced more challenging modern flight operations. In particular, satellite navigation systems, such as GPS and on-board computers that are often equipped with software-driven altitude models are used to facilitate such complex or specialized flight operations. However, the

Josaphat A. Uvah, Ph.D., professor, Department of Mathematics \& Statistics, University of West Florida. 
software-driven altitude models used for these modern flights demand that operators input data that are approximations of measurements obtained from the same mathematical model that had guided the traditional model for decades before the advent of modern technology. Consequently, the degree of accuracy in these new models depends in large measure on the original model assumptions. Therefore, it is of the essence to examine those model assumptions with the aim of developing a framework for new models that are tailored specifically for dynamical and real-time implementation, for greater precision in modern aviation.

In this paper, we examine the ISA. Among other issues, we identify the theoretical basis for its development to include the basic physical laws and the main assumptions. Aside from highlighting some limitations of the ISA, we discuss requirements and challenges for dynamical altitude modeling for specialized modern flight operations whereby external input is not utilized.

\section{The ISA Model}

The ISA model, adapted by the international civil aviation organization in 1925, is one of several standard atmosphere models in current use. It is perhaps the prevalent and most popular mathematical model used to compute true altitude in the troposphere, i.e., between eight $\mathrm{km}$ and $18 \mathrm{~km}$ above mean sea level, depending on latitude, with an average of $11 \mathrm{~km}$. West (1996) noted that attempts to similarly predict barometric pressure at high altitudes using these models produce poor results that gave pressure values too low for practical use. However, it was well known (Bilitza, 1976) that the ISA model and the United States Standard Atmosphere model of 1976 developed by the committee on extension of the standard atmosphere, were virtually indistinguishable up to an altitude of $32 \mathrm{~km}$. Consequently, our discussion in the sequel is valid for both models. We emphasize that our development does not address regions of higher altitudes, such as the stratosphere, mesosphere, and thermosphere, which require a different set of considerations.

\section{ISA Model Idealization}

Among the many considerations, the ISA model makes the following major assumptions:

1. Gravity $(g)$ is constant throughout the troposphere;

2. The temperature in the troposphere is a linear function of altitude.

A fact from elementary physics concerning gravity is that acceleration due to gravity varies with altitude. However, the first assumption is reasonable, because experimental values show small variations in the value of gravity at different altitudes but the differences are negligible in comparison to the average value of the same quantity in the troposphere (Byrd, 2007; Mahoney, 2005b; West, 1996). Geophysicists, applied physiologists, aviation experts, and other researchers all agree that air temperature varies significantly with altitude. Attempts to address these variations have led to the construction of various model atmospheres for different latitudes and seasons of the year (West, 1996). Consequently, the second assumption pertaining to the change in air temperature with altitude is very critical. In the sequel, we shall show that the second assumption accounts for a disproportionate percentage of the error in this model.

\section{ISA Model Equations}

The relationship between barometric pressure and altitude may be established from two physical laws, namely, the hydrostatic equation (also referred to as the principle of hydrostatic balance) and the ideal or perfect gas law. The hydrostatic equation is given by 


$$
d P=-\rho \mathrm{g}(z) d z
$$

where $P$ is pressure, $\rho$ is density, $z$ is the geometric height, and $g(z)$ is acceleration due to gravity at height $\mathrm{z}$.

One form of the ideal gas law relates the volume $(V)$ of air, the absolute temperature $(T)$ of the air, and its mass $(m)$ by the equation $P V=m R T$, where $R$ is the gas constant. Thus,

$$
P=\rho R T
$$

From (1) and (2), it is easy to see that

$$
P=P_{0} e^{-\int \frac{g(z)}{R T} d z}
$$

These relationships have several implications. In particular, (3) implies that if the temperature remains constant, barometric pressure would decrease exponentially with increases in altitude. On the one hand, the air temperature decreases as the altitude increases. However, the temperature of air at sea level and the rate of decrease in temperature with increases in altitude both depend on the latitude of the location, the season of the year, and other weather conditions that present disturbances (from equilibrium) in the atmosphere. In fact, West (1996) contended that these differences in temperature solely accounted for the variations in the relation between pressure and altitude among different regions of the world. Thus, the second assumption in the ISA model is a major assumption since it is based on an average to be applied to all locations, latitudes, and seasons.

The concept of geopotential altitude $(h)$ is a measurement of height relative to gravitational equipotentials, i.e., level surfaces of constant gravitational energy per unit mass (Mahoney, 2005a; 2005b). Physically, the geopotential height is defined to approximate the assumption that the acceleration due to gravity at any height remains the same as that at sea level. However, Olson (2002) had shown that at $15,240 \mathrm{~m}$, the geopotential altitude differed from the true altitude by as much as $61 \mathrm{~m}$. It is to be noted that the conversion from one altitude measure to another is possible with very high accuracy (Mahoney, 2005b).

The acceleration due to gravity $g(z)$, the earth's radius $(r)$, and gravity at the surface of the earth $g_{s}$ are related by the inverse square gravity law as follows:

$$
g(z)=g_{s}\left(\frac{r}{r+z}\right)^{2}
$$

Similarly, the relation (Olson, 2002) connected the geopotential height $h$ and the true altitude $z$ :

$$
g(z) d z=g_{s} d h
$$

Thus, while a unit of the true altitude is absolute and independent of the height, geopotential altitude decreases linearly with height, with the corresponding decrease in gravity. From (1) and (5), we obtain

$$
\frac{d P}{P}=-\frac{g_{s}}{R T} d h
$$

As discussed above, the ISA model's first assumption that gravity is constant. The ideal gas constant $R$ is also fixed. However, the temperature $T$ depends on several variables to include the true altitude height $(z)$, a positional variable latitude $(l)$, the season $(y)$, and atmospheric disturbances, such as storms $(d)$. Thus, in reality, $T$ 
$=T(z, l, x, y, d)$. In view of the relation $z=z(h)$, we observe that $T=T(h, l, x, y, d)=T(z, l, x, y, d)$, accordingly. Consequently, without further simplifying assumptions as in the ISA, (6) culminates into a complex partial differential equation that may not be readily tractable. Even if such partial differential equation were tractable, two problems are evident:

1. Formulating a correct model would be extremely involving;

2. Utility of the model for the purpose of effecting dynamical true altitude in real time will be limited due to the complexity of computations that would be needed with each set of inputs.

It is with these considerations that the ISA model's second assumption, that the temperature is a linear function of the altitude becomes an appealing alternative. Thus, if $T_{0}$ is the temperature at sea level, and the rate of decrease in temperature, known as the temperature lapse rate, is $\alpha$, then

$$
T=T_{0}-\alpha h
$$

We apply the first assumption with $g_{s}=g$ (constant), substitute (7) into (6) and solve the resulting equation, to obtain

$$
P=P_{0}\left[\frac{T_{0}-\alpha h}{T_{0}}\right]^{\frac{g}{\alpha R}}
$$

where $P_{0}$ is the pressure at sea level where $h=0$. Equation (8) establishes the pressure $P$ as a function of the geopotential altitude $(h)$, hence of the true altitude $(z)$. It is routine to see that

$$
h=\frac{T_{0}}{\alpha}\left[1-\left(\frac{P}{P_{0}}\right)^{\frac{\alpha R}{g}}\right]
$$

A graphical representation of the relationships among pressure, altitude, and temperature is shown in Figure 1 below.

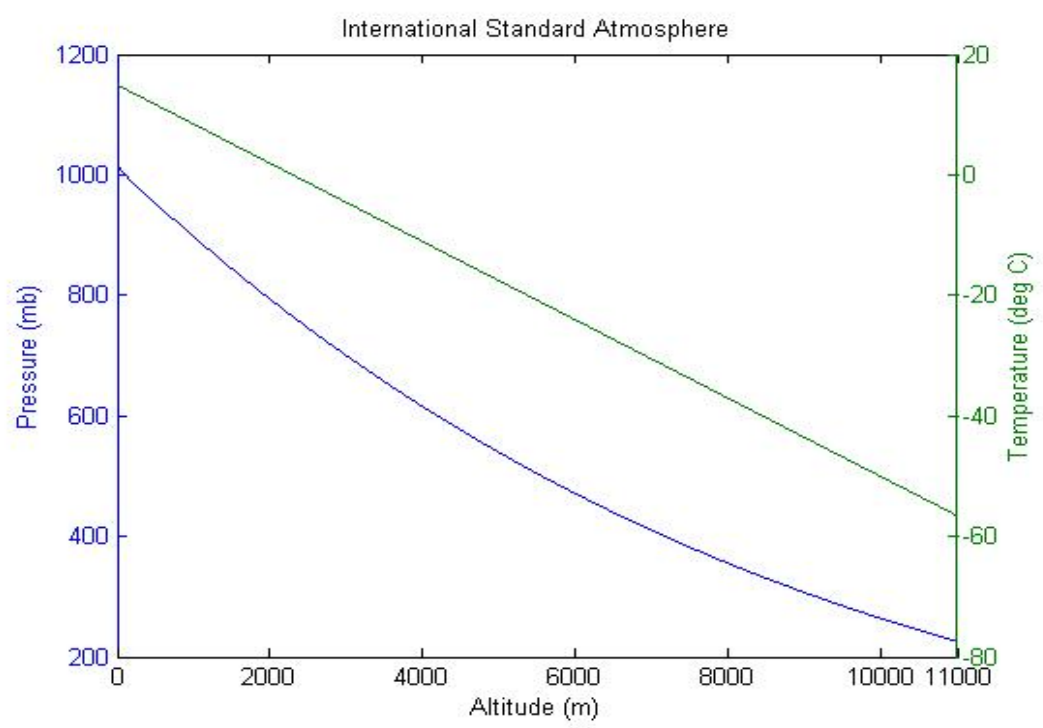

Figure 1. Pressure altitude temperature relationship in ISA Model. (Source: Byrd, 2007) 


\section{Pressure Altitude and the Barometric Altimeter}

The ISA model is designed to accommodate standard values in (9). Using these standard values, a theoretical plane is defined, the standard datum plane, where the atmospheric pressure is $1,013.25 \mathrm{mb}$ and the temperature is $15{ }^{\circ} \mathrm{C}$. The standard datum plane is the zero elevation level in the ISA model (Air Education and Training Command [AETC], 2001). The height above the standard datum plane in the ISA model is called pressure altitude, and the barometric altimeter is used to measure pressure altitude. The barometric altimeter is calibrated to the standard atmosphere and is designed to convert pressure to an altitude reading (see Figure 2).

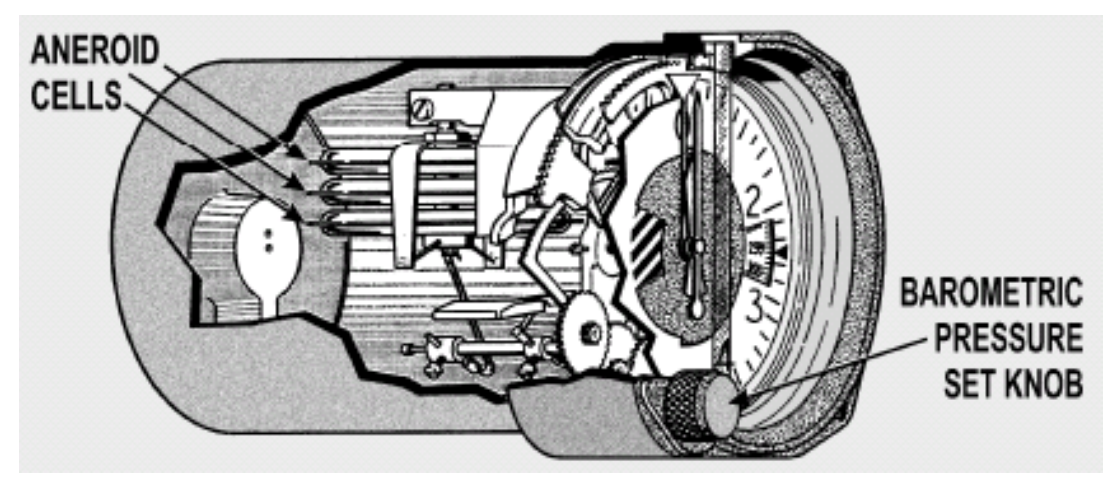

Figure 2. Barometric altimeter. (Source: Olson, 2002)

Since the standard atmosphere exists only when all initial conditions and assumptions in the ISA model are met, pressure altitude rarely corresponds to actual height above mean sea level, or true altitude. Therefore, pressure altitude is primarily used as a standard reference system and to vertically separate aircraft flying at higher altitudes (i.e., 5,486 m), where separation via a common reference is important, and separation from terrain or other obstructions is not an immediate factor. All aircraft above 18,000 feet achieve this separation by setting $1,013.25 \mathrm{mb}$ in their altimeters using the barometric pressure set knob and flying an assigned pressure altitude. The standard datum plane is not an adequate reference plane for takeoff and landing operations, because the barometric altimeter must provide an accurate altitude reading in relation to the ground, rather than the theoretical standard datum plane. This is achieved by a reading called indicated altitude. Indicated altitude is also obtained from (9) using actual values instead of standard values. Thus, as noted in (AETC, 2001), the altimeter setting is a correction for nonstandard surface pressure and should only be considered valid in close proximity to, and near the surface of, the airfield reporting that altimeter setting. However, indicated altitude does not account for nonstandard surface temperature or lapse rate. Graphically, indicated altitude is a vertical shift of the pressure altitude curve such that the origin coincides with the airfield elevation. For instance, the altimeter setting calculated for the radiosonde balloon observation (University of Wyoming, 2009) on February 1, 2005 at Sable Island, Nova Scotia, was 1,024.2 mb (see Figure 3).

By using the pressure altitude in combination with indicated altitude, routine aviation operations are performed in a safe and effective manner. However, beyond allowing for vertical traffic separation at high altitudes and localized terminal area operations near the surface, the altitude information being provided by these two types of altitude readings is not accurate. Since the initial conditions are idealized, there is the potential for errors in pressure altitude. As noted earlier, indicated altitude corrects only for nonstandard surface pressure. Nonstandard surface temperature has a major impact on the distribution of pressure layers in the atmosphere. The relationship between pressure and temperature is established in the ideal gas equation and 
the effect of surface temperature in well-known (Sanders, 2002). A sample observation in Figure 4 shows the errors.

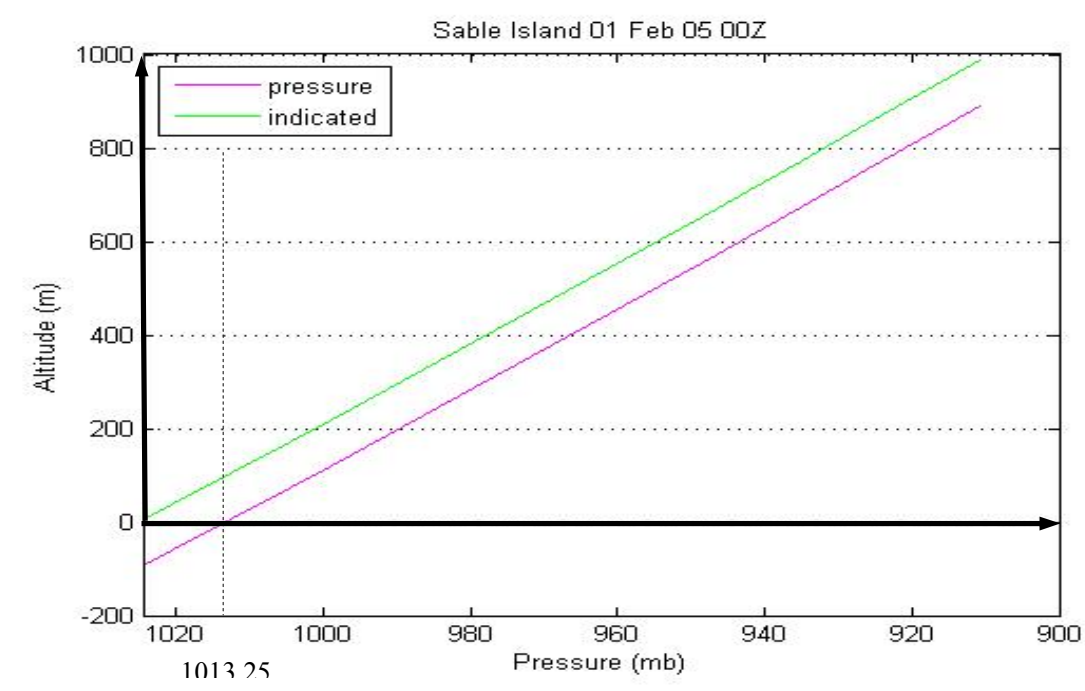

Figure 3. Pressure and indicated altitude comparison. (Source: Byrd, 2007)
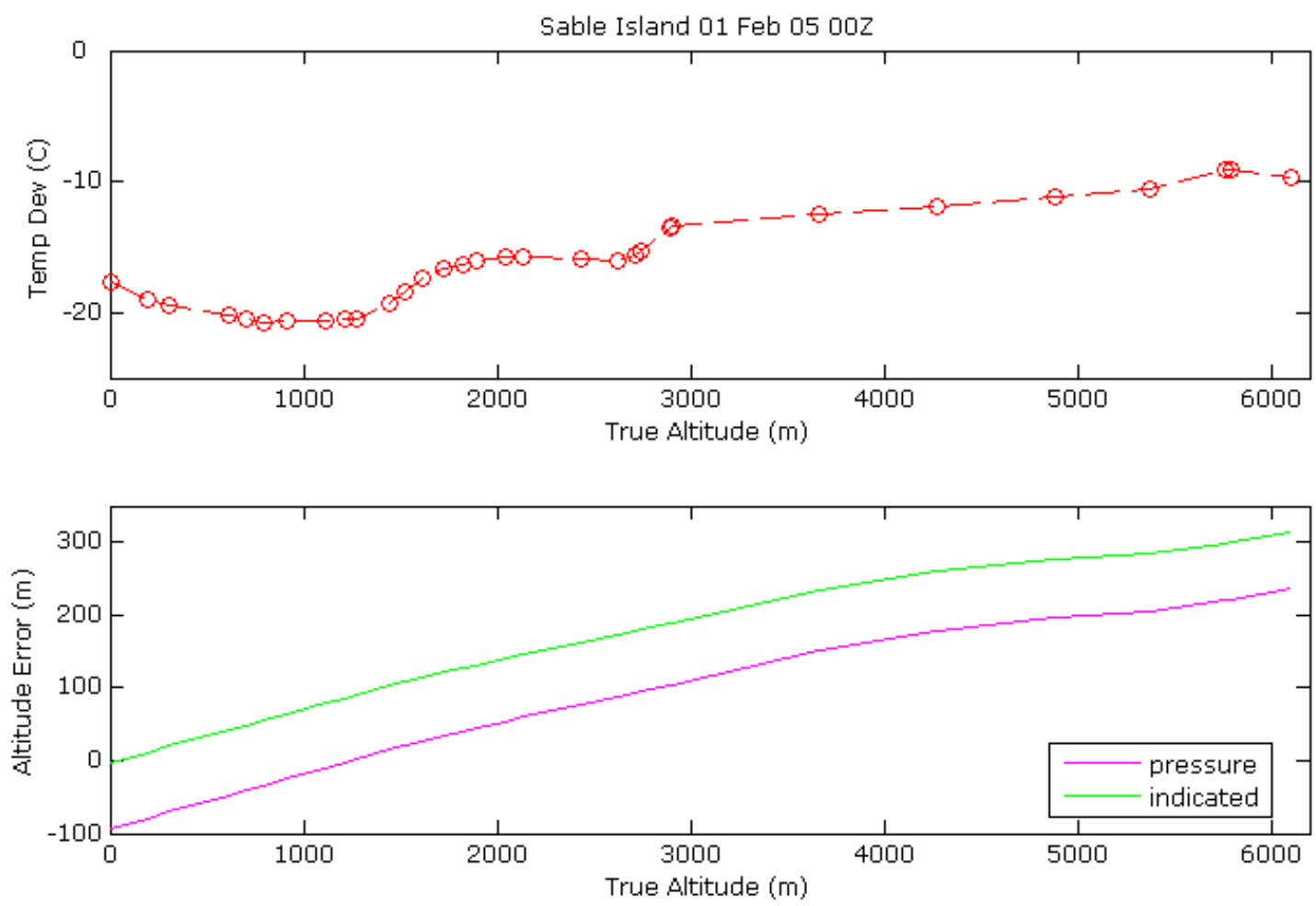

Figure 4. Temperature deviation and altitude errors. (Source: Byrd, 2007)

\section{Charles' Law and the Hypsometric Equation}

The hypsometric equation relates the geopotential thickness between two pressure levels directly to the mean virtual temperature. It is given by 


$$
Z_{1}-Z_{2}=\frac{R T_{v}}{g} \ln \left(\frac{P_{1}}{P_{2}}\right)
$$

where $Z_{i}$ are two heights with corresponding pressures $P_{i}$ and $T_{v}$ is the virtual temperature. This equation is used by weather facilities in the post-processing of atmospheric data collected by radiosonde balloons (National Oceanic and Atmospheric Administration [NOAA], 1997). Since radiosonde balloons collect pressure, temperature, and relative humidity, among other variables, ground station computer algorithms can calculate geopotential height of a pressure level as the summation of iterations of (10) from the surface to the pressure level of interest. While the hypsometric equation does not directly output an altitude above mean sea level like the ISA model, the theory and assumptions used in its derivation play an important role in our dynamical true altitude model development.

Charles' Law, also referred to as Gay-Lussac's Law, states that for a fixed mass of gas at constant pressure, the volume of the gas is directly proportional to its absolute temperature (Wallace \& Hobbs, 1977). That is, for two columns of air under constant pressure and having the same base area, where $Z$ is height of the column and $T$ is average absolute temperature in the column, the relation $\frac{T_{1}}{Z_{1}}=\frac{T_{2}}{Z_{2}}$ holds. We shall extend an application of Charles' Law to our proposed dynamical altitude model. Perhaps, Charles' law is a basis for a common rule of thumb named in several references, including the primary United States air force publication (AETC, 2001) is to compute true altitude by adjusting indicated altitude using the $2 \%$ per $5.5^{\circ} \mathrm{C}$ correction.

\section{A Framework for Dynamical Altitude Modeling}

In order to develop a framework for dynamical real time altitude modeling, we re-examine the requirements and limitations in utilizing current methods. Based on current technology and altitude modeling algorithms, the following guidelines for dynamical true altitude models are evident:

1. True altitude calculations should be performed at a rate comparable to the aircraft computer's corresponding digital pressure altitude computations;

2. Operator-initiated input of a new initial condition should be accepted always, i.e., "true" altitude, which restarts model calculations from that point. It is the operator's responsibility to ensure that the source(s) used to derive the new initial condition are providing accurate data at the time of input;

3. Operate self-contained in the temporary or complete absence of external inputs, such as GPS signals, ground-based radar data, and/or local altimeter settings provided by ground personnel;

4. Produce error rates that are acceptable for the employment regime of the aircraft in which it is utilized. This may be complex and sometimes subjective;

5. Precise temperature reading must be available at the current position of the aircraft.

However, the following factors may be limiting to the development:

1. GPS input may be absent;

2. Current surface altitude being flown over may not be known, particularly at the continuous rate required for calculations in the dynamical model;

3. Indicated altitude may not be available since it cannot be assumed that an altimeter setting is available at the surface altitude for the current or any previous position; 
4. Relative humidity is not calculated onboard most aircraft, so relative humidity inputs could only come from forecasts or actual readings from external sensors at fixed locations (e.g., a weather station at an airport).

\section{Conclusion}

It is clear from above that the linearity assumption of the temperature lapse rate accounts for many of the errors associated with true altitude modeling. Such errors are compounded when dynamical real time models are to be developed. In fact, measuring the lapse rate accurately on a given day and a fixed location may be problematic. In order to model true altitude in real time, it is conjectured that aircraft crew must constantly refine the altitude profile at short intervals, inputting new data (initial conditions) during each iteration. It would be necessary also to expand the table of temperatures (Wallace, \& Hobbs, 1977) that currently exists for only five northern latitudes $-5,30,45,65$, and 75 degrees, to a more representative data covering each region of interest. There is potential to utilize Charles' Law and the hypsometric equation to effect computations of "small" quantities involved in order to produce dynamical true altitude models.

\section{References}

Air Education and Training Command (AETC). (2001). Air navigation. Air Force Pamphlet, 11-216. Retrieved June 4, 2009, from http://www.e-publishing.af.mil/pubfiles/af/11/afpam11-216/afpam11-216.pdf

Bilitza, D. K. (1976). United States standard atmosphere. Retrieved June 30, 2009, from http://modelweb.gsfc.nasa.gov /atmos/us_standard.html

Byrd, J. V. (2007). Dynamical true altitude modeling: Theory, sufficiency, and accuracy of current and proposed models (An unpublished thesis, the University of West Florida).

Mahoney, M. J. (2005a). A brief discussion of aviation altitude scales. Retrieved June 2, 2009, from http://mtp.jpl.nasa.gov /notes/altitude/altitude.html

Mahoney, M. J. (2005b). A discussion of various measures of altitude. Retrieved June 1, 2009, from http://mtp.jpl.nasa.gov /notes/altitude/altitude.html

National Oceanic and Atmospheric Administration (NOAA). (1997). Rawinsonde and pibal observations. Federal Meteorological Handbook, 3. Retrieved June 3, 2009, from http://www.ofcm.gov/fmh3/text/

Olson, W. M. (2002). Aircraft performance flight-testing. Technical Information Handbook (AFFTC-Tlh-09-01). California: Air Force Flight Test Center. Retrieved June 1, 2009, from http://www.edwards.af.mil/sharing/tech_pubs/Handbook-10 March02.pdf

Sanders, C. (2002). Atmospheric pressure and altimeters. The Front, 1(5). Retrieved June 17, 2009, from http://aviationweather.gov/general/pubs/front/docs/oct-02.pdf

University of Wyoming. (2009). Retrieved July 12, 2009, from http://weather.uwyo.edu/upperair/sounding.html

Wallace, J. M., \& Hobbs, P. V. (1977). Atmospheric science: An introductory survey. Retrieved July 10, 2009, from http://www.atmos.washington.edu/ justinjw/PCC587/homepage_files/

West, J. B. (1996). Prediction of barometric pressures at high altitude with the use of model atmospheres. Journal of Applied Physiology, 81, 1850-1854. 